\title{
Assessment of upper extremity movement performance of patients with obstetric brachial plexus palsy
}

DOI 10.1515/cdbme-2017-0001

\begin{abstract}
Coping strategies of patients with obstetric brachial plexus palsy (OBPP) are highly individual. Up to now, individual movement performance is assessed by visual observations of physicians or therapists - a procedure, which is highly subjective and lacks objective data. However, objective data about the individual movement performance are the key to evidence-based and individualized treatment. In this paper, a new approach is presented, which provides objective information about the upper extremity movement performance of patients with OBPP. The approach is based on the use of accelerometers in combination with a classification procedure. The movement performance of 10 healthy volunteers and 41 patients with OBPP has been evaluated by experienced physiotherapists and has been assigned to one of 4 categories representing the Mallet Scale (MS) IV to I. Three triaxialaccelerometers were placed at chest, upper arm and wrist of the affected side of the patient. Acceleration signals have been recorded during repetitive movements with relevance regarding daily life. Here, especially the results from the "hand to mouth" task are presented. From the 9 recorded acceleration signals 13 relevant features were extracted. For each of the 13 features 4 thresholds have been determined distinguishing best between the 4 patient categories of the MS and the healthy subjects. With respect to the thresholds each feature value has been assigned to the discrete numbers $0,1,2,3$ or 4. Afterwards, each discrete number
\end{abstract}

\footnotetext{
*Corresponding author: Ligia C. S. Fonseca, Department of Rehabilitation \& Prevention Engineering, Institute of Applied Medical Engineering, RWTH Aachen University, Pauwelsstraße 20, 52074 Aachen Germany, E-mail: fonseca@ame.rwth-aachen.de Annika K. Nelke and Catherine Disselhorst-Klug: Department of Rehabilitation \& Prevention Engineering, Institute of Applied Medical Engineering, RWTH Aachen University, Pauwelsstraße 20, 52074 Aachen Germany, E-mail: nelke.annika@t-online.de (A. K. Nelke), disselhorst-klug@ame.rwth-aachen.de (C. Disselhorst-Klug)

Jörg Bahm: Reconstructive Surgery Unit, St. Franziskus Hospital, Morillenhang 27, 52074 Aachen Germany, E-mail: j.bahm@franziskus-hospital.de
}

has been weighted by a factor regarding the correlation between the feature's value and the MS score. The resulting weighted discrete numbers of all 13 features have been added resulting in a score, which quantifies the individual upper extremity movement performance. Based on this score the movement performance of each patient has been assigned to the classes "very good", "good", "regular" and "bad". All movements of the 10 healthy volunteers were classified as "very good". The movement performance of two patients MS IV were classified as "very good" as well and the movements of the other 16 patients as "good". The movements of the entire group of MS III patients fell into the class "regular". Just one MS II patient was assigned to the class "regular" while the others were classified as "bad". It was not possible to classify the movements of MS I patients. This was mainly due to the fact that none of these patients MS I was able to complete the task successfully. The developed approach demonstrated its ability to quantify the movement performance of upper extremity movements based on accelerometers. This provides an easy to use tool to assess patient's movement strategies during daily tasks for diagnosis and rehabilitation.

Keywords: accelerometers; birth injury; dystocia.

\section{Introduction}

Obstetric brachial plexus palsy (OBPP) as a result of an obstetric emergency and occurs in approximately 0.5 to 0.77 out of 1000 newborns $[1,2]$. In the case of OBPP the upper brachial plexus nerves are damaged and control of arm muscle function is lost. Although the nerve recovers spontaneous, arm function is severely limited in some patients [3]. Since the location and extend of the lesion of the Plexus Brachialis nerve are different in each child, the coping strategies of the patients are highly individual. Due to the development of the children with age, OBPP patients' arm function has to be regularly examined in order to agree about new therapeutic goals or to decide about operative interventions. However, up to now this is done 
mainly through visual observations by the physician or the therapist. This practice is highly subjective and lacks on objective data, which are needed to ensure comparability and individualized, evidence-based treatment. In order to achieve at least some objectivity and to document functional changes of shoulder and arm the Mallet scale (MS) is often used [4]. The MS distinguishes five different categories of severity based on specific movements. These categories are graded on a scale from I to $\mathrm{V}$, with I being not testable and IV reaching the same endpoint as healthy subjects. MS V is reserved for health subjects only. However, since reaching a certain endpoint determines the membership to a particular category, coping strategies and compensational movements are not taken into consideration.

3D movement analysis based on 3D tracking systems allows the objective analysis of upper extremity movements [2]. However, this method is complex, expensive and translation into clinical practice often fails. Nowadays accelerometers are used increasingly frequent to determine movement performance. The problem herewith is that no information about the sensor's position can be derived from the accelerometer signals directly. This is why further processing is needed to derive meaningful information about the movement performance from the sensor signals.

Based on this context, the aim of the work is to present a new approach based on the use of accelerometers in combination with a classification procedure, which allows the quantitative assessment of upper extremity movement performance of patients with OBPP. The methodology should provide direct feedback about the quality of the movement to patients, physicians and therapists and enable individualized treatment.

\section{Methods}

\subsection{Detection of movement}

Three triaxial-accelerometers (BMA180, Bosch) have been positioned at the manubrium of the sternum $\left(\mathrm{S}_{\text {chest }}\right)$, on the distal part of the upper arm $\left(\mathrm{S}_{\mathrm{arm}}\right)$ and on the wrist between distal end of ulna and radius $\left(S_{\text {hand }}\right)$. The sensors were attached direct to the skin of the subjects using a doublesided tape. Care was taken with the sensors to ensure to be at the same position. However, analysis to be applied to the signals has to cope with low deviation in position. $\mathrm{S}_{\text {chest }}$ was orientated with the X-axis turned towards the caudal direction. $S_{\text {hand }}$ and $S_{\text {arm }}$ were positioned on the affected arm of the patient with the Y-Axis parallel to the

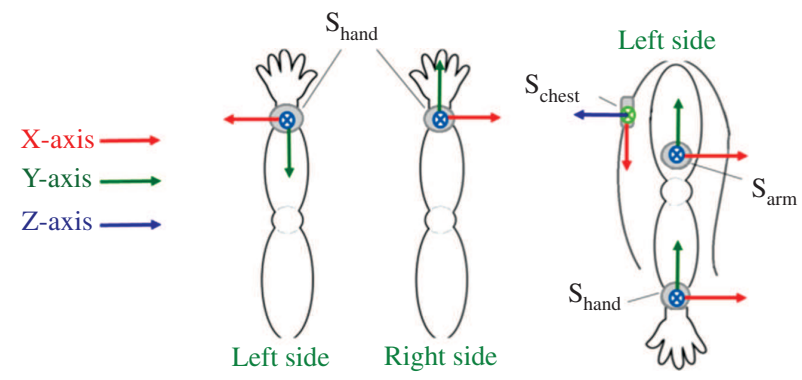

Figure 1: Position and 3D orientations of the accelerometers.

bony structures of the corresponding arm segment. Cables have been arranged in such a way that they did not hamper the movement of the patient (Figure 1).

\subsection{Subjects}

Measurements were performed on a group of 10 healthy volunteers $(M S=V)$ and 41 OBPP patients. Patients have been evaluated by an experienced physiotherapist and were assigned to one of the 4 categories of the MS (MS IV: $n=18$; MS III: $n=7$; MS II: $n=8$ and MS I: $n=8$ ). Patients were asked to perform movements representing daily activities at a comfortable speed repetitively (No. of repetitions 12). Each movement cycle started with the patient's arm in neutral position and finished after coming back to starting position.

\subsection{Features extraction}

Prior to feature extraction, movement cycles were determined on the basis of the Y-axis of the wrist sensor. From the nine acceleration signals initially 34 different features were extracted considering distribution of peaks, skewness, autocorrelation, ratios, means and standard deviations.

Peaks: Assuming that a shaky motion causes more peaks in the measured signal per cycle compared to flowing physiological movements, three different features regarding the number of positive peaks occurring in one movement cycle were estimated for each sensor separately. First, the absolute value of the acceleration was calculated as a function of time. Afterwards, the feature "mean number of peaks" was determined by averaging the number of peaks of all movement cycles. Additionally, to identify fatigue effects, the feature "change of mean number of peaks with fatigue" was calculated by subtracting the mean number of peaks of all 12 cycles from the mean number of peaks 
of the last four cycles. Furthermore, the feature "change of mean number of peaks by learning” was computed by subtracting the mean number of peaks of all the 12 cycles from the mean number of peaks of the first 4 cycles.

Skewness: The skewness of a set of samples associated with the detected signal describes the symmetry of their distribution. The sensor data was processed in the same way as described in the peaks section. Afterwards, skewness was calculated for each sensor. It results in three features for each sensor: "mean skewness", change of mean skewness with fatigue" and "change of mean skewness by learning”.

Autocorrelation: To determine the regularity of the performed movement the autocorrelation function was determined for each axis of each sensor separately. The feature "autocorrelation" is defined as the difference between the maximum of the autocorrelation function and the next occurring minimum.

Y-axis analysis: Visual inspection of the signals has shown that the different movements are best represented in the Y-axes of the different sensors. This is why some features have been chosen regarding the mean signal amplitude calculated from the Y-axes of the three sensors only. To avoid effects related to whole body movements the mean amplitude of each sensor has to be normalized. When calculating the features "Y_Chest_Mean"; " $Y_{-}$Arm_Mean" and " $Y \_$Hand_Mean" the mean amplitude of the Y-axis of each sensor was normalized to the averaged amplitude of the Y-axes of all three sensors. To compute the features "Y_Arm_Chest" and "Y_Hand_Chest" the mean amplitude values of the Y-axis of the arm and the hand sensor were normalized to the mean value of the Y-axis of the chest sensor. Finally the feature "Y-axis difference between arm and hand" was used for the analysis as well.

Standard deviation of the cycle: The feature "standard deviation of the averaged cycle duration" was used as a feature describing the regularity of the movement in time.

\subsection{Identification of relevant features and regard of their sensitivity}

A linear correlation test between the classification of the subjects with respect to the MS and the feature value was made for each feature separately. In this way, the number of $\mathrm{F}$ relevant features was identified, which are able to quantify the movement performance. A feature was accepted as suitable if the correlation coefficient was higher than 0.5. Secondly, six weighting factors $\left(\mathrm{w}_{i}: 0.5,1,1.5,2\right.$, 3 and 4) were introduced and assigned to each relevant feature. Assignment was done with respect to the strength of the correlation between the MS score and the feature value.

\subsection{Score}

To calculate a score quantifying the movement performance of each subject, four thresholds were identified for each relevant feature. The target for optimization of the thresholds was the best discrimination between the five categories of the MS. With respect to the thresholds, each feature value was assigned to a discrete number $\left(N_{i}\right)$ with 0 representing a feature value within the feature specific thresholds of MS V (healthy subjects), 1 of MS IV, 2 of MS III, 3 of MS II and 4 of MS I (most affected subjects).

An overall score (SC) quantifying the subjects' individual movement performance was calculated by adding the weighted discrete numbers of all relevant features according to:

$$
\mathrm{SC}=\sum_{i=1}^{F} w_{i} \cdot N_{i}
$$

with $F=$ number of relevant features, $w_{i}=$ weighting factor of each relevant feature and $N_{i}=$ discrete number of each feature. Classification of the patients was done on the basis of the obtained SC values. The existence of four classes "very good" (SC 0-14), "good" (SC 15-32), "regular" (SC 3345) and "bad" (SC higher 45) was assumed. Specific SC values of each class have been identified by discriminant analysis.

\section{Results}

Although different activities were investigated, here, only the results of the task "hand to the mouth" are shown.

\subsection{Relevant features}

Altogether, $F=13$ features showed a correlation higher than 0.5 between the feature value and the MS category assigned to each subject. These 13 relevant features are listed in Table 1 together with the feature's specific weighting factor $w_{i}$ and the thresholds defining the assigned discrete number $N_{i}$. 
Table 1: Comparison of the results between the groups according to the category of the Mallet Scale (MS) from V to I.

\begin{tabular}{|c|c|c|c|c|c|c|}
\hline Relev. feature & $w_{i}$ & MS V, $n=0$ & MS IV, $n=1$ & MS III, $n=2$ & MS II, $n=3$ & $M S I, n=4$ \\
\hline$F_{1}$ & 0.5 & $\infty-7.3$ & $7.3-5$ & $5-2.5$ & $2.5-\infty$ & - \\
\hline$F_{2}$ & 1 & $\infty-0.1$ & $0.1-0.4$ & $-0.4-\infty$ & - & - \\
\hline$F_{3}$ & 1.5 & $0-0.3$ & $0.3-0.5$ & $0.5-0.8$ & $0.8-\infty$ & - \\
\hline$F_{4}$ & 0.5 & $\infty-0.1$ & $0.1-0.0$ & $0.0-0.1$ & $0.1-\infty$ & - \\
\hline$F_{5}$ & 1 & $-\infty-0.1$ & $-0.1-0.0$ & $0.0-0.2$ & $0.2-\infty$ & - \\
\hline$F_{6}$ & 1.5 & $\infty-0.5$ & $0.5-0.4$ & $0.4-0.3$ & $0.3-\infty$ & - \\
\hline $\mathbf{F}_{7}$ & 2 & $-\infty-0.06$ & $0.07-0.1$ & $0.1-0.2$ & $0.2-\infty$ & - \\
\hline$F_{8}$ & 2 & $\infty-50$ & $50-40$ & $40-30$ & $30-\infty$ & - \\
\hline$F_{9}$ & 3 & $-\infty-0.1$ & $0.1-0.2$ & $0.2-0.3$ & $0.3-0.4$ & $0.4-\infty$ \\
\hline$F_{10}$ & 3 & $\infty-2.2$ & $2.2-1.9$ & $1.9-1.5$ & $1.5-0.9$ & $0.9-\infty$ \\
\hline$F_{11}$ & 3 & $-\infty-0.7$ & $0.7-1.1$ & $1.1-1.3$ & $1.4-1.5$ & $1.5-\infty$ \\
\hline$F_{12}$ & 4 & $-\infty-0.0$ & $0.0-0.2$ & $0.2-1.0$ & $1.0-2.0$ & $2-\infty$ \\
\hline$F_{13}$ & 1.5 & $-\infty-4$ & $4-5$ & 5-7 & $7-\infty$ & - \\
\hline
\end{tabular}

$\mathbf{F}_{1}=$ "mean number of peaks" $-\mathrm{S}_{\text {chest }} ; \mathbf{F}_{2}=$ "change of mean number of peaks by learning" - $\mathrm{S}_{\text {arm }} ; \mathrm{F}_{\mathbf{3}}=$ "mean skewness" - $\mathrm{S}_{\text {chest }}$; $\mathbf{F}_{4}=$ "change of mean skewness by learning" - $\mathrm{S}_{\text {arm }} ; \mathbf{F}_{\mathbf{5}}=$ "change of mean skewness with fatigue" - $\mathrm{S}_{\text {arm }} ; \mathbf{F}_{6}=$ "autocorrelation" - $\mathrm{Y}$-axis $\mathrm{S}_{\text {hand }} ; \mathbf{F}_{7}=$ "autocorrelation" - Z-axis $S_{\text {hand }} ; \mathbf{F}_{\mathbf{8}}=$ "Y_Hand_Chest"; $\mathbf{F}_{\mathbf{9}}=$ "Y_Chest_Mean"; $\mathbf{F}_{10}=$ "Y_Arm_Mean"; $\mathbf{F}_{11}=$ "Y_Hand_Mean"; $\mathbf{F}_{12}=$ " $Y$-axis difference between arm and hand"; $\mathbf{F}_{13}=$ "standard deviation of the averaged cycle duration".

\subsection{Group and individual results}

The SC score as a function of the MS category is shown in Figure 2. All participants of the heathy control group showed a SC between 0 and 14 and were assigned to the class "very good". Since this group of healthy participants forms the base-line, the class "very good" represents a physiological movement performance.

Two patients categorized as MS IV by the physiotherapists were classified as "very good" as well. However, 16 patients with MS IV were assigned to the class "good" on the basis of the SC value. All patients of the class "good" performed with light compensational movements.

All MS III patients as well as one MS II patient had a SC value between 33 and 45 and fell into the class "regular". Subjects allocated to this class show movement patterns with medium compensational parts. All other

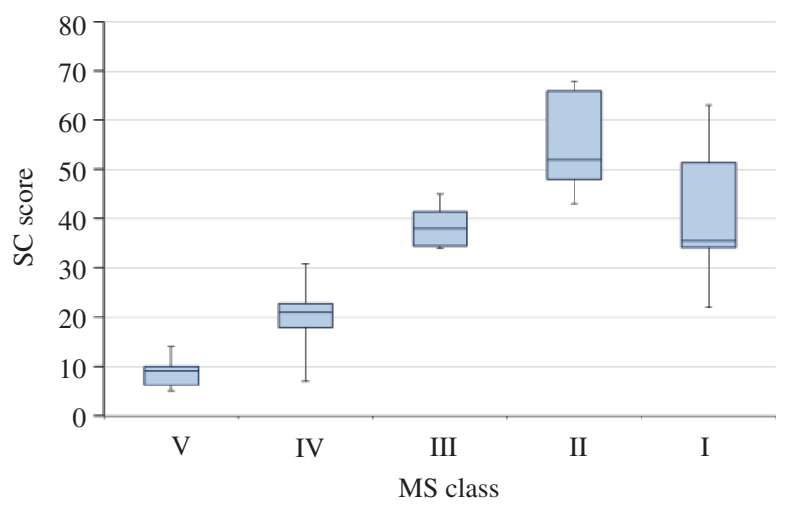

Figure 2: SC scores related to the categories of the MS. patients categorized to MS II by the physiotherapists were classified as "bad" (SC higher than 45) and needed a couple of compensational movements to perform the task.

It was not possible to assign MS I patients to a common class. Two of them fell into the class "good", three into the class "regular" and the remaining three into the class "bad". None of them was classified as "very good". In general the execution of the movement task was better in this group of patients when compared to MS IV patients, who showed more compensatory movements. Considering the facts that the category MS I includes patients being not testable because of not being able to reach any end-point and that accelerometers fail in detecting certain positions, the limitations of the developed procedure became obvious in this group. The introduced procedure is designed to evaluate movement performance and to quantify the severity of compensational movement parts. Since MS I is just defined by not reaching a certain end-point, movement performance is more heterogenic in this category compared to the other MS categories.

\section{Conclusion}

The introduced approach demonstrated its ability to assess upper extremity movement performance in patients with OBPP quantitatively. Based on the classification, direct feedback about the quality of movement performance can be given to patients, physicians and therapists. The quantitative and objective assessment of relevant movements 
for daily activities will enable an evidence-based, individualised treatment for OBPP patients.

Acknowledgment: The authors thank CAPES for the support of the work. (Coordination for the Improvement of Higher Education Personnel under grant number 11924-13-6).

\section{Author's Statement}

Research funding: The work is funded by Coordination for the improvement of higher education personnel (CAPES) under grant number 11924-13-6. Conflict of Interest: Authors state no conflict of interest. Informed consent: Informed consent has been obtained from all the individuals included in this study. Ethical approval: The research related to human use complies with all the relevant national regulations, institutional policies and was performed in accordance with the tenets of the Helsinki Declaration, and has been approved by the authors' institutional review board or equivalent committee.

\section{References}

[1] Silva S, Mimoso G, Araújo H, Gonçalves O. Paralisia do plexo braquial no recém nascido: experiência de dez anos numa maternidade de apoio perinatal diferenciado. Acta Pediátrica Portuguesa 2010;41:127-30.

[2] Bahm J, Meinecke L, Brandenbusch V, Rau G, Disselhorst-Klug C. High spatial resolution electromyography and video-assited movement analysis in children with obstetric brachial plexus palsy. Hand Clin. 2003;19:393-9.

[3] Bahm J, Uphoff R, Mahler M. Der geburtstraumatische Plexus brachialis Schaden: Information für Betroffene, interessierte Laien und Fachleute. UIm: s.n; 2010.

[4] Blaauw G, Muhlig RS. Measurement of external rotation of the shoulder in patients with obstetric brachial plexus palsy. J Brachial Plex Peripher Nerve Inj. 2012;7:8. 